\title{
Neutrophil Extracellular Traps and Its Correlation with Several Pathological Conditions: Prosperities and Deleterious Implications
}

\author{
Phey Liana ${ }^{1,2}$, Krisna Murti ${ }^{3}$, Zen Hafy ${ }^{4}$, Iche Andriyani Liberty ${ }^{5}$, Tungki Pratama Umar ${ }^{6}$ \\ ${ }^{1}$ Department of Clinical Pathology, Faculty of Medicine, Universitas Sriwijaya/Dr Mohammad Hoesin General Hospital, \\ Palembang, Indonesia \\ ${ }^{2}$ Biomedicine Doctoral Program, Faculty of Medicine, Universitas Sriwijaya, Palembang, Indonesia \\ ${ }^{3}$ Department of Anatomic Pathology, Faculty of Medicine, Universitas Sriwijaya, Palembang, Indonesia \\ ${ }^{4}$ Biomedical Department, Faculty of Medicine, Universitas Sriwijaya, Palembang, Indonesia \\ ${ }^{5}$ Department of Public Health and Community Medicine, Universitas Sriwijaya, Palembang, Indonesia \\ ${ }^{6}$ Charitas Hospital KM 7, Palembang, Indonesia
}

\begin{abstract}
Neutrophil extracellular traps (NETs) are immune components found in a variety of pathological states. It has been shown to have either beneficial or harmful implications, depending on how it is controlled and has been particularly observed in three major scenarios: infection, autoimmune disease, and cancer. In this article, we compiled some of the roles of NETs in pathological conditions, as well as the benefits of targeting them for improved patient outcomes. The role of NETs were primarily positive in infectious disease, whether caused by bacteria, virus, or fungal infection. In non-infectious inflammatory scenarios, on the other hand, it's the complete opposite, with the effects being mainly deleterious and even worse than the original disease states. Targeting NETs directly or indirectly may help to prevent complications and improve patient outcomes. A plethora of compounds, including immunomodulators, anti-thrombosis, nicotinamide adenine dinucleotide phosphate (NADPH)/reactive oxygen species (ROS) inhibitors, nuclease, and other compounds, may be used to accomplish the therapeutic goals.
\end{abstract}

Keywords: autoimmune disease, cancer, DNase, infection, neutrophil extracellular traps

\section{Introduction}

Neutrophils, also known as polymorphonuclear leukocytes (PMN), are the most plentiful leukocytes in the blood involved in the pathogen defensive system. ${ }^{1}$ Several activities, including the fabrication of neutrophil extracellular traps
(NETs), extracellular webs made up of nuclear and granular parts of the cell that arise from decondensed chromatin, have been discovered per its action. ${ }^{2}$ The formation of NETs (NETosis) for limiting and removing external infections is a different approach in comparison with necrosis and apoptosis. $^{3-5}$ In many circumstances, bacteria, fungi, and

Date of submission: October 28, 2021

Last Revised: November 14, 2021

Accepted for publication: November 24, 2021

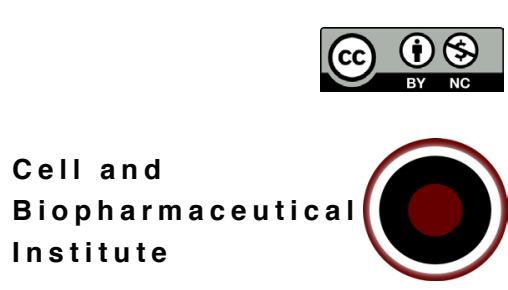

Corresponding Author:

Zen Hafy

Biomedical Department, Faculty of Medicine, Universitas Sriwijaya

Jl. Dokter Muhammad Ali, Sekip Jaya, Palembang, Indonesia

e-mail: zenhafy@gmail.com 
viruses are susceptible to NETs, indicating that NETs play a beneficial role. ${ }^{6}$ On the contrary, a lack of proper NETs controls may result in immunothrombosis, which can have serious consequences for the patient. ${ }^{7}$

As previously stated, the role of NETs is a doubleedged sword: at certain levels and conditions, this molecule acts as a non-specific immune response component; however, under uncontrolled conditions, it can cause tissue damage, initiate inflammatory cells, and contribute to pathological processes such as systemic inflammatory response syndrome (SIRS), acute lung injury, thrombosis, autoimmune diseases, multiple sclerosis, and cancer. ${ }^{2,8-10}$ Tissue damage is mostly linked to increased levels of NETs and a reduction in DNase synthesis. ${ }^{11,12}$ Through stimulating $\mathrm{CD}^{+} \mathrm{T}$ cells, NETs play a role in the adaptive immune system. ${ }^{13}$ NETs can also activate naive $\mathrm{CD} 4^{+} \mathrm{T}$ cells and memory $\mathrm{CD}^{+} \mathrm{T}$ cells, which can then induce $\mathrm{CD} 8^{+} \mathrm{T}$ cell activation via interleukin (IL)-2, resulting in higher interferon (IFN)-1 production. ${ }^{13,14}$ Intracellular proteins are exposed to the extracellular space during NETosis and have the potential to serve as autoantigens against the host, resulting in a vicious circle. ${ }^{15}$ Thus, a greater understanding of the role of NETs and their targeting potential are required in a variety of situations, whether infectious or noninfectious disease. Regarding this condition, we briefly summarize some of NETs' roles in pathological conditions, as well as the benefits of its targeting for improving patient outcomes.

\section{NETosis}

NETosis is a kind of cell death that is distinct from necrosis and apoptosis, and its mechanism is unknown, reflecting the neutrophil's key involvement in various inflammatory conditions. ${ }^{16,17}$ Microscopic organisms, inflammation, and endogenous stimuli can all trigger this occurrence. Neutrophil signaling can lead to adhesion towards the Toll-like receptor (TLR), Fc receptors, and complement receptors, which can lead to the formation of NETs, which involve cytokines including IL-8, tumor necrosis factor (TNF), and IFN- $\gamma^{2}$

Suicidal NETosis (programmed cell death) and vital NETosis (either reactive oxygen species (ROS)-dependent or ROS-independent) are the two primary forms of NETosis. Suicidal NETosis is the most prevalent type of NETosis, which begins when neutrophils are triggered by an inductor material, the most efficient of which is phorbol 12-myristate 13-acetate (PMA), in addition to lipopolysaccharides
(LPS), or calcium ionophores (CaI) ${ }^{18}$ Neutrophil activation would enhance protein kinase $\mathrm{C}$ activity, resulting in nicotinamide adenine dinucleotide phosphate (NADPH) oxidase (NOX) engagement in the plasma and granular membranes. After that, chromatin decondensation is undertaken., ${ }^{45,19-21}$ Several enzymes, such as neutrophil elastase (NE), myeloperoxidase (MPO), and peptidyl arginine deiminase 4 (PAD4), play an important part in this process. ${ }^{22}$ Simultaneously, vesicles formed on the nuclear membrane, producing membrane damage, while the cytoplasmic granular membrane disintegrates, allowing granular contents and chromatin to merge. ${ }^{21,23}$ After that, these elements will be liberated into the extracellular environment, and the NETosis will be completed when the plasma membrane disintegrates. ${ }^{4,20,24}$ The process is simplified in Figure 1.

The formation of NETs begins with ligand binding to neutrophils via TLRs or IgG-Fc receptors, complement or cytokines. ${ }^{3}$ Activation of neutrophils through the protein kinase C (PKC)/Raf-MEK-ERK kinase and Rac2 pathway will stimulate the NOX complex..$^{20,21,25}$ In addition, PKC isoenzymes can also inhibit histone deamination and activate the enzyme PAD4 in complement with an increase in $\mathrm{Ca}_{2}{ }^{+}$in the cytosol. PAD4 causes histone hipercitrullination which results in chromatin decondensation (by weakening DNAhistone electrostatic bonds). ${ }^{21,26,27}$ In addition to PAD4, chromatin decondensation is aided by NE and MPO. MPO focuses on expanding chromatin condensation while $\mathrm{NE}$ degrades $\mathrm{H} 1$ and processes histone nuclei. The molecular key to the establishment of NETs is PAD4-dependent $\mathrm{Ca}^{+}$ citrullination of histone $\mathrm{H} 3{ }^{20}$

ROS-dependent vital NETosis is associated with neutrophil priming using the granulocyte-macrophage colony-stimulating factor (GM-CSF). GM-CSF is activated by LPS or C5a and releasing mitochondrial DNA simultaneously with granular proteins that depend on ROS production. ${ }^{2,15,21,28}$ The involvement of ROS that can be considered as a signaling molecule rather than the cytotoxic antimicrobial activity has implications in the formation of NETs. ${ }^{4}$ Meanwhile, the ROS-independent condition of vital NETosis is linked to the rapid release of nuclear DNA while maintaining the nuclear neutrophil function, allowing them to complete the phagocytosis and classical bacterial killing process. ${ }^{29}$ All vital NETosis processes allow neutrophils to remain active and capable of carrying out phagocytosis and consist of growth of the nuclear envelope, decondensation of chromatin, and destruction of the nuclear membrane. ${ }^{24}$ 


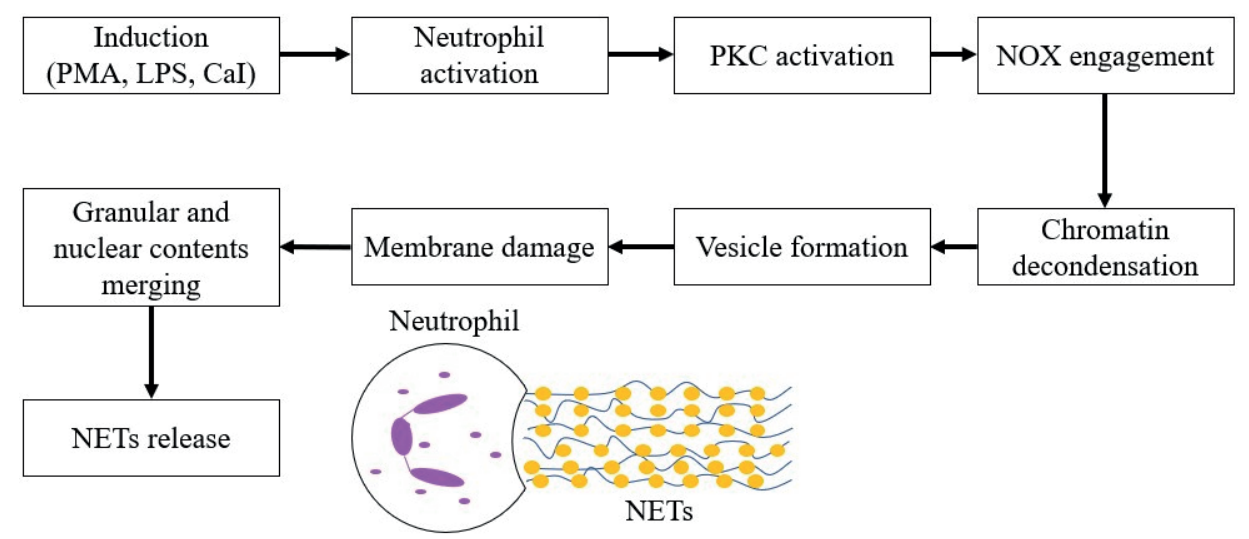

Figure 1. NETs formation (NETosis).

\section{NETs role in non-specific (innate) immune response system and its control of release}

As pointed out previously, NETs are sourced from neutrophils, which are cellular components of non-specific immune systems (along with macrophage, mast cell, and dendritic cell). Its function in innate immune systems is related to the trapping of pathogenic microbes, which prevents microorganism spread, limits organ damage, and increases the concentration of released microbicidal agents in a specified target. ${ }^{30}$ NETs can also cooperate with B and $\mathrm{T}$ lymphocytes, communicating the innate and adaptive immune systems and encouraging their activation (via NET/cell interaction and TCR signaling). ${ }^{13,31}$ Excessive NETs secretion (which is linear with neutrophil expansion) can, on the contrary, lead to adverse disease progression, such as worse clinical manifestations, a higher likelihood of clinical instability, a longer length of hospital stay, and increased 30-day all-cause mortality events. ${ }^{32,33}$ As a result, the good or bad aspect of NETs are reliant on sustaining a rigid balance of its release and removal. ${ }^{2}$

Following the preceding statement, NETs control and regulation must be implemented to avoid negative consequences. Although the precise molecular mechanisms regulating NETs formation are still largely unknown, several mediators have been identified to play a role. The primary regulatory compounds are NE and MPO which is activated by ROS, causing NETosis to occur. NE and MPO then migrated to the nucleus. NE cleaves histones and encourages chromatin decondensation, whereas MPO binds to chromatin later, promoting further decondensation. NE and MPO can also conspire to promote chromatin decondensation, resulting in cell destruction and NETs release. ${ }^{34}$ Other regulatory compounds are PAD4 (mediator of histone citrullination), nicotinamide adenine dinucleotide phosphate/NADPH (inhibitor of PMA-induced NETs formation), mammalian target of rapamycin (mTOR), protein kinase A (PKA), and PKC..$^{35}$ The signaling pathways that regulate NET formation varied depending on the stimulant and warrants further investigation. ${ }^{36}$ The summary regarding NETs role in innate immune system and its control is depicted in Figure 2.

\section{NETs role in several pathological conditions}

\section{Infection}

NETs have been demonstrated to have a role in a variety of infections, including those caused by viruses, bacteria, protozoa, helminths, and fungal invasion, among others..$^{37-40}$ The role was mainly positive, such as killing microbes directly or interacting with numerous antimicrobial peptides, such as histones and granule-resident antimicrobial proteins (NE, MPO, Gasdermin D (GSDMD), cathepsin G, $\alpha$-defensins, and lactoferrin), which have been studied extensively in bacterial infection. ${ }^{41,42}$ Microbial entrapment, toxin degradation, and virulence factors breakdown, also played a role in the struggle against the infection. ${ }^{43}$ Some ions, like iron and zinc, may interact with NETs to prevent microbial proliferation. ${ }^{44}$

\section{Virus}

Viral infections, including the Chikungunya, Human Immunodeficiency Virus (HIV), Dengue, Respiratory Syncytial Virus (RSV), and Influenza have been associated with the NETs. Beneficial effects of NETs have been observed to promote viral neutralization which is dependent to the TLR7 (an endolysosome able to recognize single- 


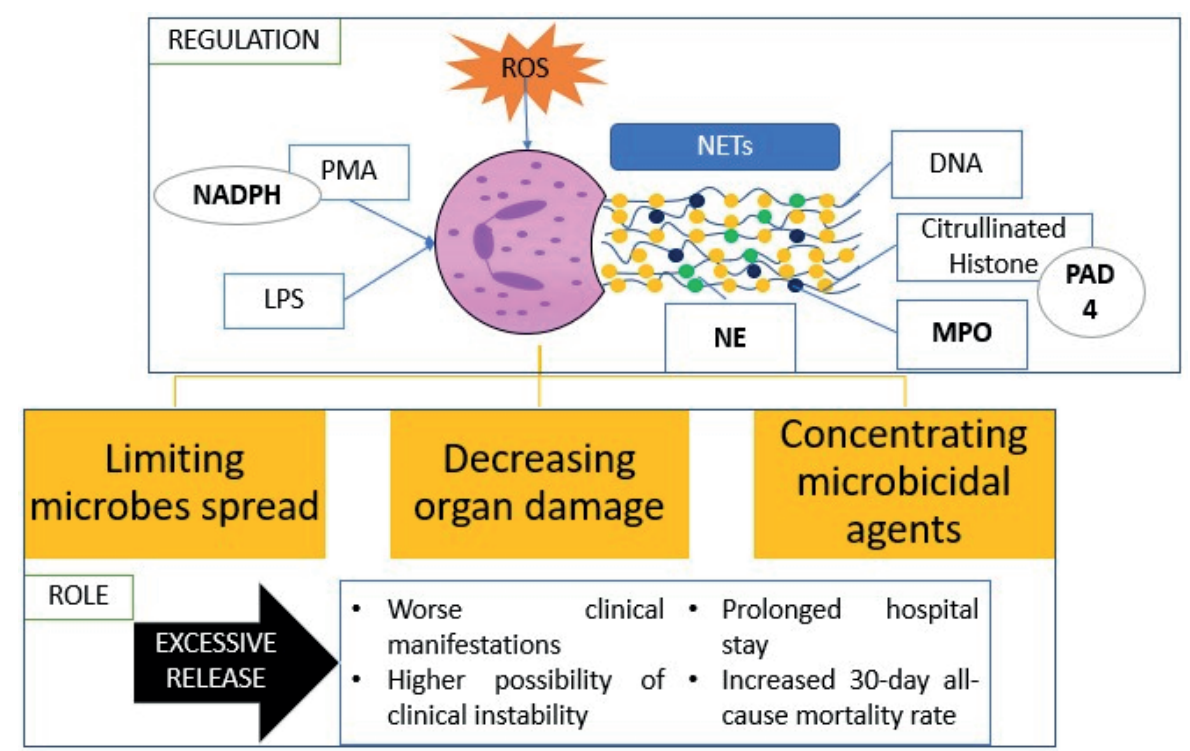

Figure 2. The role of NETs in the innate immune system and its control particles. NETs have three main mechanisms that are responsible for cellular protection; however, excessive release can result in some negative effects, as shown in the illustration. Several regulatory compounds have been identified and are linked to a variety of inductor proteins, including NADPH (for PMA), PAD4 (for citrullinated histone), NE, and MPO (for ROS). The regulatory compounds are denoted by words in bold type.

stranded RNA virus) activation and ROS production. The virus has been inactivated by the aggregation with the free DNA and citrullinated histone $\mathrm{H} 3$ (H3cit) which is responsible for capturing the virus particles in the studies simulating Chikungunya virus..$^{45}$ A study also concluded that NETs were able to inhibit HIV-1 proliferation in macrophage, which is responsible for almost $80 \%$ reduction without affecting the survivability of the macrophage. The inhibition of viral replication mostly caused by the interference in HIV-1-DNA integration, an early critical step in viral reproducibility, in addition to causing decreased viral fitness and facilitating macrophage production of $\beta$-chemokine. ${ }^{46}$ In the study which is assessing the protective effect of NETs to the RSV, it is concluded that low concentration of NETs were beneficial to counteract the virus. It is hypothesized to be associated with direct viral capturing or viral neutralization through the interaction with the myeloperoxidase, histones, defensins. But, in high concentration, NETs were responsible for massive cellular damage (which is greater than the virus effect) ${ }^{47}$

However, although NETs have provided some beneficial effects in countering the viral infection, it is also potential to induce some detrimental effects. In the RSV infection, although NETs have been considered to prevent viral infection and its binding to the epithelial cell, it is also able to induce airway obstruction which is leading into severe features of the disease. ${ }^{48}$ The high level of NETs level in the plasma was also correlated with the worse features of the disease caused by the infection of Influenza virus. Acute Lung Injury (ALI), which was caused by the interaction between Influenza virus and NETs was associated with increased alveolar epithelial protein permeability. ${ }^{49}$ Dengue virus, on the other hand, was able to induce NETs formation, which is responsible for promoting pathogenic effects, increased vascular permeability, an important mechanisms for the progression of severe dengue infection. ${ }^{50}$

NETs-associated biomarkers, histone DNA (hisDNA) complexes, and NETs-specific MPO-DNA complexes were discovered to be markedly elevated in plasma samples and/or tracheal aspirates from a critically ill COVID-19 patient population (including those who undergo mechanical ventilation). ${ }^{51,52}$ As an outcome, an increase in NETs concentration is related to a worsening of the disease, which can be observed through imaging studies or autopsies..$^{51,52}$ The association of NETs with at least seven acute-phase protein genes (IL6, TNF, CRP, CXCL8, IL-1ß, IL-17A, and IL-1) was also aligned with disease deterioration. ${ }^{53}$ In severe COVID-19 contexts, 
several pathological mechanisms that underlie the poorer outcome include a hyperinflammatory state (cytokine storm), immunothrombosis, and microvascular endothelial injury, which may also be arbitrated by NETs. ${ }^{54}$

\section{Bacteria}

NETs role mainly observed in sepsis cases. It has been determined to promote the Escherichia coli capturing, which is originating from the platelet TLR4 activation. ${ }^{55}$ The intravascular NETs were responsible for acute protection of E. coli invasion to the liver cells and hematogenous spread of the bacteria to the distant organs. The histones are also responsible for the immuno-neutralization activity, leading into reduced organ damage and enhanced survival in the sepsis and endotoxemia cases. However, some pathological findings have been observed, which is correlated with the hepatic damage, induced by NETs. ${ }^{56}$ In the observation using the Staphylococcus aureus, it was found that NETs, in companion with MPO have significant effect in bacterial killing, when combined with hydrogen peroxide $\left(\mathrm{H}_{2} \mathrm{O}_{2}\right)$. It is mainly related to the oxidative damage produced by $\mathrm{H}_{2} \mathrm{O}_{2}$ activity, which is a two-sided phenomenon, either beneficial or detrimental. ${ }^{57}$

As stated before, NETs have potential benefits and also some harmful effects in the battle against bacterial infection. For the example, a study was assessing Pseudomonas aeruginosa-associated keratitis. It was found that NETs were responsible for preventing $P$. aeruginosa dissemination to the brain by the dead-zone formation, which is related to the type-3secretion system (T3SS). However, prevention of brain dissemination poses worse pathological effect to the eye and antimicrobial resistance by the biofilm formation. 58 In the Ventilator-associated pneumonia cases, NETs, measured as MPO-DNA complexes, are highly detected in alveolar space of the critically ill patients which is associated with greater bacterial burden and neutrophilic inflammation process. This process could lead into Acute Respiratory Distress Syndrome (ARDS) which is negatively affect patient's prognosis. ${ }^{59}$ Another case in pneumococcal meningitis have shown that NETs did not causing any reduction in bacterial viability. The bacteria was able to disperse into other organs and also the brain. Dissemination of the bacteria to the brain was related to an increased bacterial load and Blood Brain Barrier (BBB) leakage. ${ }^{60}$

\section{Fungal}

Candida albicans was the most commonly observed fungal organism in determining NETs function. C. albicans infestation is reliant on the ability of the organism to switch between cellular and hyphal (even pseudohyphal) forms, which is fundamental for the transformation from candidemia to tissue invasion. ${ }^{61-63}$ Phagocytosis can remove cellular (blastoconidia) forms, but hyphal forms are too big to be ingested, interfering this characteristic antimicrobial host protective mechanism. Thus, an alternative defensive method, NETs, could play a role in the battle against C. albicans (in both cellular and hyphal forms). ${ }^{64}$ The involvement of NETs in regulating complement receptor type 3 (CR3) in modulating the host defenses to fungal pathogen-associated molecular patterns (PAMP), which is dependent on the extracellular signal-regulated kinase/ mitogen-activated protein kinase (ERK/MAPK) pathway, was significant. ${ }^{65}$ Unopsonized C. albicans elicits NOXindependent NETosis via dectin-2 and its downstream signaling pathway (Syk-Ca2 $2^{+}-\mathrm{PKC} \delta$-PAD4/NE), which aids in limiting fungal dissemination and transmission to the subsequent organs, according to a study. ${ }^{66}$ NETs are also triggered by various $C$. albicans components, including the principal cell wall-building polysaccharides (mannans and $\beta$-glucans) and secreted aspartic proteases (Saps). Sap4 and Sap6, which are proven to be released by fungal hyphae via ROS-dependent ( $\beta$-Glucans) and ROS-independent (Mannans) signaling pathways, notably through linkages with the $\mathrm{CD} 11 \mathrm{~b}$ receptor, are the most potent NET-releasing responses. ${ }^{67}$

Although NETs are substantially effective in combating fungal infection, C. albicans has developed some defensive schemes to resist their effects. The extracellular matrix-mediated inhibitory pathway produced by $C$. albicans biofilms may impede neutrophil response, resulting in impaired NETs release and diminished fungal termination. ${ }^{6}$ Furthermore, the production of DNAse I or catalase, as well as cytochalasin D, may dramatically decrease NET function, allowing C. albicans to evade NETs capturing and killing. ${ }^{69}$

\section{Autoimmune Disease}

Systemic Lupus Erythematosus (SLE), Rheumatoid Arthritis (RA), psoriasis, and antiphospholipid syndrome are all autoimmune conditions in which NETs play an important role. ${ }^{70,71}$ Proteins located in NETs, such as MPO and proteinase 3 (PR3) was hypothesized as the source of essential autoantigens. ${ }^{72}$ Anti-myeloperoxidase and anti-protein 3 in anti-neutrophil cytoplasmic antibodies (ANCA)-associated vasculitis (AAV), anti-double-stranded DNA (dsDNA) in SLE, anti-citrullinated protein antibodies 
(ACPAs) in RA, and human cathelicidin LL-37 in psoriasis have all been found to play an autoantigenic position. ${ }^{73,74}$ Production of ROS and its interplay with pro-inflammatory cytokines such as TNF- $\alpha$, IL-1, IL-8, and IL-17 are thought to produce NET formation. ${ }^{75}$ NETs have been shown to have a major impact on the severity of autoimmune disorders and to hasten their progression ${ }^{73,76}$ due to chemicals existing in the NETs or NETs degradation product by DNAse I which operating as auto-antigens, or due to overwhelming NET degradation failure ${ }^{77}$.

As stated above, NETs have been considered as the source of autoantigens in SLE. ${ }^{78,79}$ NETs production have been associated with low-density granulocyte (LDG) activity which significantly correlated with the cell-free DNA (cfDNA) concentration, a biomarker of active SLE. ${ }^{80}$ In the lupus nephritis patient, in addition to increased cfDNA concentration, higher level of quantitative 24-hour urinary protein and suppression of creatinine clearance rate also found. ${ }^{78}$ Neutrophils from SLE patients with active disease may induce the release of NETs, resulting in overexpression of the stress-related protein regulated in development and DNA damage response 1 (REDD1). SLEassociated NETs were decorated with the tissue factor (TF) and IL-17A, which pioneered thrombin production and the fibrotic process in discoid skin lesions, glomerular and tubulointerstitial compartments. ${ }^{81}$ In addition, lower ability of SLE patient's serum to degrade NETs may be associated with severe disease feature, attributed to an activation of complement systems, mainly involved $\mathrm{C} 3$ and $\mathrm{C} 4 .^{82}$

In the patients with rheumatoid arthritis, NETs posed both pro and anti-inflammatory effects. A study have shown that it is modulated by the $\mathrm{Clq}$ and cathelicidin (LL37). ${ }^{83}$ The anti-inflammatory effects were attributed by the inhibition of IL- 6 secretion and increased secretion of the immunomodulatory cytokine IL-10. Meanwhile, proinflammatory states were shown by the increase in IL-8 levels and up-regulation of CD11b. ${ }^{83}$ Histopathological examination have shown that NETs may disrupt articular cartilage by its elastase activity which induces the release of membrane-bound peptidylarginine deiminase-2 by fibroblast-like synoviocytes (FLSs), in addition to activation of macrophage. ${ }^{84}$ Targeting of NETs may be beneficial for RA treatment, which has been examined in a study using $\mathrm{Cl}$-amidine, a highly specific inhibitor of PAD4. Inhibition of NETs release significantly attenuated collagen-induced arthritis (CIA) and suppressed the stimulation of antigenbearing dendritic cells (DCs). ${ }^{85}$
An increase of NETs level also observed in psoriasis patients. Its increase were related to the progressing disease severity. A model study stated that NETs, which is formed through the mast cells and neutrophils were responsible for an increased expression of IL-17. ${ }^{86}$ It may provide a biologic basis for systemic features of psoriasis, and increase in atherosclerosis and mortality. ${ }^{87,88}$ But, another study also suggested direct role of NETs in the activation of inflammatory response in keratinocytes, by involving the TLR4/IL-36R crosstalk. ${ }^{89}$ NETs also able to create a vicious cycle of skin inflammation by making the complex with LL37 triggered TLR8/TLR13-mediated cytokine. ${ }^{74}$ However, it can induce the production of human $\beta$-defensin- 2 (HBD-2), an antimicrobial peptide which is overexpressed in epidermal keratinocyte of psoriasis patients. By this mechanisms, it can help in protecting the psoriasis plaque from being infected by secondary bacterial infections. ${ }^{88}$ Inhibition of NETs, either by CI-amidine or DNase I was beneficial to decrease scaling, acanthosis, and inflammatory infiltrate in histopathological study of psoriatic skin. ${ }^{89}$

\section{Cancer}

Neutrophils and NETs have been understood to have a role in tumor growth. NETs were solely accountable for tumor progression and proliferation, as well as distant metastasis. $^{90,91}$ Inflammation-induced awakening of quiescent cancer cells may be mediated via NETs and NETmediated extracellular matrix (ECM) alteration. NETs can awaken cancer cells, allowing for successive proteolytic remodeling of laminin and integrin-mediated signaling in the tumor cells. ${ }^{92}$ In addition to promoting tumor angiogenesis, hypercoagulation (through a rise in endogenous thrombin potential), and cancer-associated thrombosis, NETs were found to hinder cytotoxic lymphocytes from assaulting cancer cells. ${ }^{93-95}$ Upon the stimulation of the ILK- $\beta$-parvinRAC1-CDC42 pathway, which can improve cell motility, the transmembrane protein CCDC25 was assumed to guide the metastatic process. ${ }^{96,97}$ However, because of the neutrophil's antitumor and protumor (N1 and N2) capabilities, NETs may play a role in tumor formation or inhibition, depending on the context, position, and volume generated ${ }^{98}$

NETs have been observed to poses significant association with the breast cancer stages. It has been found that the higher levels of NE-DNA complexes were examined in regional and distant stages as compared with localized disease. ${ }^{99}$ NETs also poses some detrimental phenotypes which is strongly associated with pro-metastatic features of the cancer cells. NETs were able to induce the alteration of 
morphology dan migratory pattern of the cancer cell line (MCF7), intensifying epithelial-mesenchymal transition (through cadherin switching), modifying the expression pattern of solid tumor stem cell markers, CD24 and CD44, in addition to intercalating pro-inflammatory markers, including IL-1 $\beta / I L 1 B$, IL6, and IL-8/CXCL8, markers. All of these features collaborate to produce a more aggressive breast cancer subtypes. ${ }^{100}$ A study have shown that PAD4 and nuclear factor kappa-B (NF- $\mathrm{B}$ ), which activated by the NETs were responsible for inducing inflammation and metastasis of the cancer cells. Application of NEMObinding domain (NBD) for suppressing NETs were useful for preventing lung metastasis in mice model of breast cancer. ${ }^{101}$

In the colorectal cancer patients, NETs may play a direct tumor proliferative role and inhibit tumor cell apoptosis. The involvement of several factors, including the exosomes, IL-8, and mast cells may take a role in metastatic process, especially in the liver, lung, and peritoneum. ${ }^{102,103}$ Procoagulant phenotype also promoted by excessive platelet-activated NETs release, involving the changes in human umbilical vein endothelial cells (HUVECs). It shows an increased risk of venous thromboembolism, as evidenced by significant correlation with thrombin-antithrombin (TAT) complexes and D-dimer. ${ }^{104} \mathrm{~A}$ study also evaluated higher NETs production in patients with significant complications following colorectal cancer operation (Clavien Dindo classification $\geq 3$ ). The differences also found in the patients with longer length of stay ( $>5$ days) and died, all of which with higher NETs level. ${ }^{105}$ Following surgery, immune response will be activated and have an important role for reparative processes besides promoting the risk of tumor recurrence and distant metastasis. ${ }^{106}$ However, in another study, tumor resection was observed to suppressed NETs formation, reduced apoptosis, and elevated phagocytosis. ${ }^{107}$ The effect is not only limited for colon surgery, but also the surgery for metastatic cells in the liver. Interaction between NETs, high mobility group box 1 (HMGB1) and TLR9 activation following surgery was responsible for protumorigenic properties and tumor progression. Thus, the targeting of DNAse and PAD4 may inhibit these events. ${ }^{108}$

\section{Implication for targeting NETs as therapeutic approach in several pathological conditions}

As previously stated, NETs have favorable impacts in a variety of infectious conditions; however, conflicting evidence exists on their impact in some non-infectious inflammatory disorders, such as autoimmune disease and cancer, leading to the conclusion that there are "bad" and "good" NETs. ${ }^{109}$ The role of NETs in various pathological circumstances, as well as NETs control via drugs that either impair NETs formation or target NETs components such as DNA or neutrophil proteases, is still being investigated. ${ }^{110}$ DNAse administration was effective in preventing cancer metastasis ${ }^{11}$, decreasing thrombotic complications ${ }^{34}$, promoting wound healing and preventing scar formation ${ }^{112}$, in addition to inhibiting activation of alternative complement pathway and endothelial damage ${ }^{113,114}$.

Another strategy involving thrombomodulin was found to be protective against endothelial disorder in sepsis and have a favorable therapeutic capacity for sepsisinduced coagulopathy. ${ }^{115}$ Thrombomodulin also hampered the induction of NETs and suppressed the epithelial to mesenchymal transition in cancer cells by degrading high mobility group box protein 1 (HMGB1), thus inhibiting cancer metastasis. ${ }^{116,117}$ The use of recombinant humansoluble TM (rTM) can also completely inhibit NETosis, inhibiting nucleic acid and NETs-mediated coagulation both in vivo and in vitro. ${ }^{118,119}$

In summary, four mechanisms can be used to target NETs for therapeutic purposes: (1) NETs induction blockade (CXCR-ligands, IL-8, and G-CSF); (2) NETs formation pathway inhibition (NOX2, NE, MPO, or PAD4); (3) NETs structure destruction using DNase; and (4) NETstumor contact impediment (integrin, TLR9, or CCDC25). ${ }^{120}$ Anti-inflammatory/immunomodulatory, anti-thrombosis, NADPH/ROS inhibitors, nuclease, and other substances (including probiotics and vitamin D) are among the antiNETs treatment modalities. Hypercoagulation intervention, oxidative stress reduction, NETosis inhibition, endothelial damage minimization, and cell apoptosis decrease are among the primary priorities of the NETs inhibition. ${ }^{121}$

\section{Conclusion}

Depending on the pathological states, NETs have both positive (primarily for infectious disease) and negative (for non-infectious disease) implications. As a result, to target the disease more specifically, it is necessary to carefully understand the types of stimulus and the signaling pathways in every pathological process, which requires more mechanistic and clinical study. Thus, NETs can be used as a potential marker for predicting patient prognosis and outcome. By directly targeting NETs destruction or 
indirectly targeting NETs induction and formation, several adverse events that are even worse than the natural course of the disease can be prevented.

\section{References}

1. Rosales C. Neutrophil: a cell with many roles in inflammation or several cell types? Front Physiol. 2018; 9: 113. doi: 10.3389/ fphys.2018.00113.

2. Kaplan MJ, Radic M. Neutrophil extracellular traps: double-edged swords of innate immunity. J Immunol. 2012; 189(6): 2689-95.

3. Yang H, Biermann MH, Brauner JM, Liu Y, Zhao Y, Herrmann M. New insights into neutrophil extracellular traps: mechanisms of formation and role in inflammation. Front Immunol. 2016; 7: 302. doi: 10.3389/fimmu.2016.00302.

4. Papayannopoulos V, Zychlinsky A. NETs: a new strategy for using old weapons. Trends Immunol. 2009; 30(11): 513-21.

5. Kobayashi Y. Neutrophil biology: an update. EXCLI J. 2015; 14 : $220-7$.

6. Lu T, Kobayashi SD, Quinn MT, Deleo FR. A NET Outcome. Front Immunol. 2012; 3: 365. doi: 10.3389/fimmu.2012.00365.

7. Zhang H, Zhou Y, Qu M, Yu Y, Chen Z, Zhu S, et al. Tissue factorenriched neutrophil extracellular traps promote immunothrombosis and disease progression in sepsis-induced lung injury. Front Cell Infect Microbiol. 2021; 11: 677902. doi: 10.3389/ fcimb.2021.677902.

8. Ramirez GA, Manfredi AA, Rovere-Querini P, Maugeri N. Bet on NETs! Or on how to translate basic science into clinical practice. Front Immunol. 2016; 7: 417. doi: 10.3389/fimmu.2016.00417.

9. Mitsios A, Arampatzioglou A, Arelaki S, Mitroulis I, Ritis K. NETopathies? Unraveling the dark side of old diseases through neutrophils. Front Immunol. 2017; 7: 678. doi: 10.3389/ fimmu.2016.00678.

10. Franchi T, Eaton S, De Coppi P, Giuliani S. The emerging role of immunothrombosis in paediatric conditions. Pediatr Res. 2019; 86(1): 19-27.

11. Meng W, Paunel-Görgülü A, Flohé S, Witte I, Schädel-Höpfner $\mathrm{M}$, Windolf $\mathrm{J}$, et al. Deoxyribonuclease is a potential counter regulator of aberrant neutrophil extracellular traps formation after major trauma. Mediators Inflamm. 2012; 2012: 149560. doi: $10.1155 / 2012 / 149560$.

12. Yousefi S, Simon D, Stojkov D, Karsonova A, Karaulov A, Simon HU. In vivo evidence for extracellular DNA trap formation. Cell Death Dis. 2020; 11(4): 300. doi: 10.1038/s41419-020-2497-x.

13. Tillack K, Breiden P, Martin R, Sospedra M. T lymphocyte priming by neutrophil extracellular traps links innate and adaptive immune responses. J Immunol. 2012; 188(7): 3150-9.

14. Garcia-Romo GS, Caielli S, Vega B, Connolly J, Allantaz F, Xu Z, et $a l$. Netting neutrophils are major inducers of type I IFN production in pediatric systemic lupus erythematosus. Sci Transl Med. 2011; 3(73): 73ra20. doi: 10.1126/scitranslmed.3001201.

15. Jorch SK, Kubes P. An emerging role for neutrophil extracellular traps in noninfectious disease. Nat Med. 2017; 23(3): 279-87.

16. Fuchs TA, Abed U, Goosmann C, Hurwitz R, Schulze I, Wahn V, et $a l$. Novel cell death program leads to neutrophil extracellular traps. J Cell Biol. 2007; 176(2): 231-41.

17. Konig MF, Andrade F. A critical reappraisal of neutrophil extracellular traps and NETosis mimics based on differential requirements for protein citrullination. Front Immunol. 2016; 7: 461. doi: 10.3389/ fimmu.2016.00461.

18. Neubert E, Senger-Sander SN, Manzke VS, Busse J, Polo E, Scheidmann SEF, et al. Serum and serum albumin inhibit in vitro formation of neutrophil extracellular traps (NETs). Front Immunol. 2019; 10: 12. doi: 10.3389/fimmu.2019.00012.

19. Kumar V, Sharma A. Neutrophils: Cinderella of innate immune system. Int Immunopharmacol. 2010; 10(11): 1325-34.

20. Goldmann O, Medina E. The expanding world of extracellular traps: not only neutrophils but much more. Front Immunol. 2013; 3: 420. doi: 10.3389/fimmu.2012.00420.

21. Delgado-Rizo V, Martínez-Guzmán MA, Iñiguez-Gutierrez L, GarcíaOrozco A, Alvarado-Navarro A, Fafutis-Morris M. Neutrophil extracellular traps and its implications in inflammation: an overview. Front Immunol. 2017; 8: 81. doi: 10.3389/fimmu.2017.00081.

22. Tokuhiro T, Ishikawa A, Sato H, Takita S, Yoshikawa A, Anzai R, et al. Oxidized phospholipids and neutrophil elastase coordinately play critical roles in NET formation. Front cell Dev Biol. 2021; 9: 718586. doi: 10.3389/fcell.2021.718586.

23. Manda A, Pruchniak MP, Araźna M, Demkow UA. Neutrophil extracellular traps in physiology and pathology. Cent J Immunol. 2014; 39(1): 116-21.

24. Lauková L, Konečná B. NETosis - Dr. Jekyll and Mr. Hyde in inflammation. J Appl Biomed. 2018; 16(1): 1-9. doi: 10.1016/j. jab.2017.10.002.

25. Branzk N, Papayannopoulos V. Molecular mechanisms regulating NETosis in infection and disease. Semin Immunopathol. 2013; 35(4): 513-30.

26. Wang Y, Li M, Stadler S, Correll S, Li P, Wang D, et al. Histone hypercitrullination mediates chromatin decondensation and neutrophil extracellular trap formation. J Cell Biol. 2009; 184(2): 205-13.

27. Leshner M, Wang S, Lewis C, Zheng H, Chen XA, Santy L, et al. PAD4 mediated histone hypercitrullination induces heterochromatin decondensation and chromatin unfolding to form neutrophil extracellular trap-like structures. Front Immunol. 2012; 3: 307. doi: 10.3389/fimmu.2012.00307.

28. O'Brien XM, Biron BM, Reichner JS. Consequences of extracellular trap formation in sepsis. Curr Opin Hematol. 2017; 24(1): 66-71.

29. Tatsiy O, McDonald PP. Physiological stimuli induce PAD4dependent, ROS-independent NETosis, with early and late events controlled by discrete signaling pathways. Front Immunol. 2018; 9: 2036. doi: 10.3389/fimmu.2018.02036.

30. Warren E, Teskey G, Venketaraman V. Effector mechanisms of neutrophils within the innate immune system in response to Mycobacterium tuberculosis infection. J Clin Med. 2017; 6(2): 15. doi: $10.3390 /$ jcm6020015.

31. Grayson PC, Kaplan MJ. At the bench: neutrophil extracellular traps (NETs) highlight novel aspects of innate immune system involvement in autoimmune diseases. J Leukoc Biol. 2016; 99(2): 253-64.

32. Bartoli ML, Costa F, Malagrinò L, Nieri D, Antonelli S, Decusatis $\mathrm{G}$, et al. Sputum inflammatory cells in COPD patients classified according to GOLD 2011 guidelines. Eur Respir J. 2016; 47(3): 978-80.

33. Ebrahimi F, Giaglis S, Hahn S, Blum CA, Baumgartner C, Kutz A, et al. Markers of neutrophil extracellular traps predict adverse outcome in community-acquired pneumonia: secondary analysis of a randomised controlled trial. Eur Respir J. 2018; 51(4): 1701389. doi: 10.1183/13993003.01389-2017.

34. Papayannopoulos V, Metzler KD, Hakkim A, Zychlinsky A. 
Neutrophil elastase and myeloperoxidase regulate the formation of neutrophil extracellular traps. J Cell Biol. 2010; 191(3): 677-91.

35. Li T, Zhang Z, Li X, Dong G, Zhang M, Xu Z, et al. Neutrophil extracellular traps: signaling properties and disease relevance. Mediators Inflamm. 2020; 2020: 9254087. doi: 10.1155/2020/9254087.

36. Kenny EF, Herzig A, Krüger R, Muth A, Mondal S, Thompson PR, et al. Diverse stimuli engage different neutrophil extracellular trap pathways. Elife. 2017; 6: e24437. doi: 10.7554/eLife.24437.

37. Jenne CN, Wong CHY, Zemp FJ, McDonald B, Rahman MM, Forsyth PA, et al. Neutrophils recruited to sites of infection protect from virus challenge by releasing neutrophil extracellular traps. Cell Host Microbe. 2013; 13(2): 169-80.

38. Arazna M, Pruchniak MP, Demkow U. Neutrophil extracellular traps in bacterial infections: strategies for escaping from killing. Respir Physiol Neurobiol. 2013; 187(1): 74-7.

39. Díaz-Godínez C, Carrero JC. The state of art of neutrophil extracellular traps in protozoan and helminthic infections. Biosci Rep. 2019; 39(1): BSR20180916. doi: 10.1042/BSR20180916.

40. Urban CF, Nett JE. Neutrophil extracellular traps in fungal infection. Semin Cell Dev Biol. 2019; 89: 47-57.

41. Burgener SS, Schroder K. Neutrophil extracellular traps in host defense. Cold Spring Harb Perspect Biol. 2020; 12(7): a037028. doi: 10.1101/cshperspect.a037028.

42. Li RHL, Tablin F. A comparative review of neutrophil extracellular traps in sepsis. Front Vet Sci. 2018; 5: 291. doi: 10.3389/ fvets.2018.00291.

43. von Köckritz-Blickwede M, Blodkamp S, Nizet V. Interaction of bacterial exotoxins with neutrophil extracellular traps: impact for the infected host. Front Microbiol. 2016; 7: 402. doi: 10.3389/ fmicb.2016.00402.

44. Kuźmicka W, Moskalik A, Manda-Handzlik A, Demkow U, Wachowska M, Ciepiela O. Influence of iron-and zinc-chelating agents on neutrophil extracellular trap formation. Cent Eur J Immunol. 2021; 46(1): 135-9.

45. Hiroki CH, Toller-Kawahisa JE, Fumagalli MJ, Colon DF, Figueiredo L, Fonseca BALD, et al. Neutrophil extracellular traps effectively control acute chikungunya virus infection. Front Immunol. 2020; 10: 3108. doi: 10.3389/fimmu.2019.03108.

46. Mojoli A, Gonçalves BS, Temerozo JR, Cister-Alves B, Geddes $\mathrm{V}$, Herlinger A, et al. Neutrophil extracellular traps from healthy donors and HIV-1-infected individuals restrict HIV-1 production in macrophages. Sci Rep. 2020; 10(1): 19603. doi: 10.1038/s41598020-75357-2.

47. Souza PSS, Barbosa LV, Diniz LFA, Da Silva GS, Lopes BRP, Souza PMR, et al. Neutrophil extracellular traps possess anti-human respiratory syncytial virus activity: Possible interaction with the viral F protein. Virus Res. 2018; 251: 68-77.

48. Cortjens B, De Boer OJ, De Jong R, Antonis AFG, Sabogal Piñeros YS, Lutter R, et al. Neutrophil extracellular traps cause airway obstruction during respiratory syncytial virus disease. J Pathol. 2016; 238(3): 401-11.

49. Zhu L, Liu L, Zhang Y, Pu L, Liu J, Li X, et al. High level of neutrophil extracellular traps correlates with poor prognosis of severe influenza A infection. J Infect Dis. 2018; 217(3): 428-37.

50. Sung PS, Huang TF, Hsieh SL. Extracellular vesicles from CLEC2activated platelets enhance dengue virus-induced lethality via CLEC5A/TLR2. Nat Commun. 2019; 10(1): 2402. doi: 10.1038/ s41467-019-10360-4.

51. Veras FP, Pontelli MC, Silva CM, Toller-Kawahisa JE, de Lima
M, Nascimento DC, et al. SARS-CoV-2-triggered neutrophil extracellular traps mediate COVID-19 pathology. J Exp Med. 2020; 217(12): e20201129. doi: 10.1084/jem.20201129.

52. Ouwendijk WJD, Raadsen MP, van Kampen JJA, Verdijk RM, von der Thusen JH, Guo L, et al. High levels of neutrophil extracellular traps persist in the lower respiratory tract of critically ill patients with coronavirus disease 2019. J Infect Dis. 2021; 223(9): 1512-21.

53. Bouzid A, Uthman AT, Al-Rawi NN, Al-Rawi NH. Neutrophil extracellular traps in coronavirus infection: interaction network analysis. Syst Rev Pharm. 2020; 11(11): 1091-101.

54. Mastellos DC, da Silva BGPP, Fonseca BAL, Fonseca NP, Auxiliadora-Martins M, Mastaglio S, et al. Complement C3 vs C5 inhibition in severe COVID-19: Early clinical findings reveal differential biological efficacy. Clin Immunol. 2020; 220: 108598. doi: 10.1016/j.clim.2020.108598.

55. Clark SR, Ma AC, Tavener SA, McDonald B, Goodarzi Z, Kelly $\mathrm{MM}$, et al. Platelet TLR4 activates neutrophil extracellular traps to ensnare bacteria in septic blood. Nat Med. 2007; 13(4): 463-9.

56. McDonald B, Urrutia R, Yipp BG, Jenne CN, Kubes P. Intravascular neutrophil extracellular traps capture bacteria from the bloodstream during sepsis. Cell Host Microbe. 2012; 12(3): 324-33.

57. Parker H, Albrett AM, Kettle AJ, Winterbourn CC. Myeloperoxidase associated with neutrophil extracellular traps is active and mediates bacterial killing in the presence of hydrogen peroxide. J Leukoc Biol. 2012; 91(3): 369-76.

58. Thanabalasuriar A, Scott BNV, Peiseler M, Willson ME, Zeng $\mathrm{Z}$, Warrener $\mathrm{P}$, et al. Neutrophil extracellular traps confine Pseudomonas aeruginosa ocular biofilms and restrict brain invasion. Cell Host Microbe. 2019; 25(4): 526-36.e4.

59. Mikacenic C, Moore R, Dmyterko V, West TE, Altemeier WA, Liles WC, et al. Neutrophil extracellular traps (NETs) are increased in the alveolar spaces of patients with ventilator-associated pneumonia. Crit Care. 2018; 22(1): 358. doi: 10.1186/s13054-018-2290-8.

60. Mohanty T, Fisher J, Bakochi A, Neumann A, Cardoso JFP, Karlsson $\mathrm{CAQ}$, et al. Neutrophil extracellular traps in the central nervous system hinder bacterial clearance during pneumococcal meningitis. Nat Commun. 2019; 10(1): 1667. doi: 10.1038/s41467019-09040-0.

61. Noble SM, Gianetti BA, Witchley JN. Candida albicans cell-type switching and functional plasticity in the mammalian host. Nat Rev Microbiol. 2017; 15(2): 96-108.

62. Kornitzer D. Regulation of Candida albicans hyphal morphogenesis by endogenous signals. J Fungi. 2019; 5(1): 21. doi: 10.3390/ jof5010021.

63. Bar-Yosef H, Gonzalez NV, Ben-Aroya S, Kron SJ, Kornitzer D. Chemical inhibitors of Candida albicans hyphal morphogenesis target endocytosis. Sci Rep. 2017; 7: 5692. doi: 10.1038/s41598017-05741-y.

64. Branzk N, Lubojemska A, Hardison SE, Wang Q, Gutierrez MG, Brown GD, et al. Neutrophils sense microbe size and selectively release neutrophil extracellular traps in response to large pathogens. Nat Immunol. 2014; 15(11): 1017-25.

65. Byrd AS, O'Brien XM, Johnson CM, Lavigne LM, Reichner JS. An extracellular matrix-based mechanism of rapid neutrophil extracellular trap formation in response to Candida albicans. J Immunol. 2013;190 (8): 4136-48.

66. Wu SY, Weng CL, Jheng MJ, Kan HW, Hsieh ST, Liu FT, et al. Candida albicans triggers NADPH oxidase-independent neutrophil extracellular traps through dectin-2. PLoS Pathog. 2019; 15(11): e1008096. doi: 10.1371/journal.ppat.1008096. 
67. Zawrotniak M, Bochenska O, Karkowska-Kuleta J, Seweryn-Ozog $\mathrm{K}$, Aoki W, Ueda M, et al. Aspartic proteases and major cell wall components in Candida albicans trigger the release of neutrophil extracellular traps. Front Cell Infect Microbiol. 2017; 7: 414. doi: 10.3389/fcimb.2017.00414.

68. Johnson CJ, Cabezas-Olcoz J, Kernien JF, Wang SX, Beebe DJ, Huttenlocher A, et al. The extracellular matrix of Candida albicans biofilms impairs formation of neutrophil extracellular traps. PLoS Pathog. 2016; 12(9): e1005884. doi: 10.1371/journal.ppat.1005884.

69. Zhang X, Zhao S, Sun L, Li W, Wei Q, Ashman RB, et al. Different virulence of Candida albicans is attributed to the ability of escape from neutrophil extracellular traps by secretion of DNase. Am J Transl Res. 2017; 9(1): 50-62.

70. Goel RR, Kaplan MJ. Deadliest catch: neutrophil extracellular traps in autoimmunity. Curr Opin Rheumatol. 2020; 32(1): 64-70.

71. Lee KH, Kronbichler A, Park DDY, Park Y, Moon H, Kim H, et al. Neutrophil extracellular traps (NETs) in autoimmune diseases: a comprehensive review. Autoimmun Rev. 2017; 16(11): 1160-73.

72. Dwivedi N, Radic M. Burning controversies in NETs and autoimmunity: the mysteries of cell death and autoimmune disease. Autoimmunity. 2018; 51(6): 267-80.

73. Fousert E, Toes R, Desai J. Neutrophil extracellular traps (NETs) take the central stage in driving autoimmune responses. Cells. 2020; 9(4): 915. doi: 10.3390/cells9040915.

74. Herster F, Bittner Z, Archer NK, Dickhöfer S, Eisel D, Eigenbrod T, et al. Neutrophil extracellular trap-associated RNA and LL37 enable self-amplifying inflammation in psoriasis. Nat Commun. 2020; 11: 105. doi: 10.1038/s41467-019-13756-4.

75. Keshari RS, Jyoti A, Dubey M, Kothari N, Kohli M, Bogra J, et al. Cytokines induced neutrophil extracellular traps formation: implication for the inflammatory disease condition. PLoS One. 2012; 7(10): e48111. doi: 10.1371/journal.pone.0048111.

76. Barnado A, Crofford LJ, Oates JC. At the Bedside: Neutrophil extracellular traps (NETs) as targets for biomarkers and therapies in autoimmune diseases. J Leukoc Biol. 2016; 99(2): 265-78.

77. Navegantes KC, de Souza Gomes R, Pereira PAT, Czaikoski PG, Azevedo CHM, Monteiro MC. Immune modulation of some autoimmune diseases: the critical role of macrophages and neutrophils in the innate and adaptive immunity. J Transl Med. 2017; 15: 36. doi: 10.1186/s12967-017-1141-8.

78. Zhang S, Lu X, Shu X, Tian X, Yang H, Yang W, et al. Elevated plasma cfDNA may be associated with active lupus nephritis and partially attributed to abnormal regulation of neutrophil extracellular traps (NETs) in patients with systemic lupus erythematosus. Intern Med. 2014; 53(24): 2763-71.

79. Jeremic I, Djuric O, Nikolic M, Vlajnic M, Nikolic A, Radojkovic D, et al. Neutrophil extracellular traps-associated markers are elevated in patients with systemic lupus erythematosus. Rheumatol Int. 2019; 39(11): 1849-57.

80. Xu Y, Song Y, Chang J, Zhou X, Qi Q, Tian X, et al. High levels of circulating cell-free DNA are a biomarker of active SLE. Eur J Clin Invest. 2018; 48(11): e13015. doi: 10.1111/eci.13015.

81. Frangou E, Chrysanthopoulou A, Mitsios A, Kambas K, Arelaki S, Angelidou I, et al. REDD1/autophagy pathway promotes thromboinflammation and fibrosis in human systemic lupus erythematosus (SLE) through NETs decorated with tissue factor (TF) and interleukin-17A (IL-17A). Ann Rheum Dis. 2019; 78(2): 238-48.

82. Leffler J, Martin M, Gullstrand B, Tydén H, Lood C, Truedsson L, et $a l$. Neutrophil extracellular traps that are not degraded in systemic lupus erythematosus activate complement exacerbating the disease.
J Immunol. 2012; 188(7): 3522-31.

83. Ribon M, Seninet S, Mussard J, Sebbag M, Clavel C, Serre G, et al. Neutrophil extracellular traps exert both pro-and anti-inflammatory actions in rheumatoid arthritis that are modulated by C1q and LL37. J Autoimmun. 2019; 98: 122-31.

84. Carmona-Rivera C, Carlucci PM, Goel RR, James E, Brooks SR, Rims C, et al. Neutrophil extracellular traps mediate articular cartilage damage and enhance cartilage component immunogenicity in rheumatoid arthritis. JCI insight. 2020; 5(13): e139388. doi: 10.1172/jci.insight.139388.

85. Papadaki G, Kambas K, Choulaki C, Vlachou K, Drakos E, Bertsias $\mathrm{G}$, et al. Neutrophil extracellular traps exacerbate Th1-mediated autoimmune responses in rheumatoid arthritis by promoting DC maturation. Eur J Immunol. 2016; 46(11): 2542-54.

86. Lin AM, Rubin CJ, Khandpur R, Wang JY, Riblett M, Yalavarthi S, et al. Mast cells and neutrophils release IL-17 through extracellular trap formation in psoriasis. J Immunol. 2011; 187(1): 490-500.

87. Gelfand JM, Troxel AB, Lewis JD, Kurd SK, Shin DB, Wang X, et $a l$. The risk of mortality in patients with psoriasis: results from a population-based study. Arch Dermatol. 2007; 143(12): 1493-9.

88. Hu SCS, Yu HS, Yen FL, Lin CL, Chen GS, Lan CCE. Neutrophil extracellular trap formation is increased in psoriasis and induces human $\beta$-defensin-2 production in epidermal keratinocytes. Sci Rep. 2016; 6: 31119. doi: 10.1038/srep31119.

89. Shao S, Fang H, Dang E, Xue K, Zhang J, Li B, et al. Neutrophil extracellular traps promote inflammatory responses in psoriasis via activating epidermal TLR4/IL-36R crosstalk. Front Immunol. 2019; 10: 746. doi: 10.3389/fimmu.2019.00746.

90. Cools-Lartigue J, Spicer J, Najmeh S, Ferri L. Neutrophil extracellular traps in cancer progression. Cell Mol life Sci. 2014; 71(21): 4179-94.

91. Park J, Wysocki RW, Amoozgar Z, Maiorino L, Fein MR, Jorns $\mathrm{J}$, et al. Cancer cells induce metastasis-supporting neutrophil extracellular DNA traps. Sci Transl Med. 2016; 8(361): 361ra138. doi: 10.1126/scitranslmed.aag1711.

92. Albrengues J, Shields MA, Ng D, Park CG, Ambrico A, Poindexter ME, et al. Neutrophil extracellular traps produced during inflammation awaken dormant cancer cells in mice. Science. 2018; 361(6409): eaao4227. doi: 10.1126/science.aao4227.

93. Cristinziano L, Modestino L, Antonelli A, Marone G, Simon HU, Varricchi G, et al. Neutrophil extracellular traps in cancer. Semin Cancer Biol. 2021; 2021: S1044-579X(21)00206-6. doi: 10.1016/j. semcancer.2021.07.011.

94. Jung HS, Gu J, Kim JE, Nam Y, Song JW, Kim HK. Cancer cell-induced neutrophil extracellular traps promote both hypercoagulability and cancer progression. PLoS One. 2019; 14(4): e0216055. doi: 10.1371/journal.pone.0216055.

95. Demers M, Wagner DD. Neutrophil extracellular traps: A new link to cancer-associated thrombosis and potential implications for tumor progression. Oncoimmunology. 2013; 2(2): e22946. doi: 10.4161/ onci.22946.

96. Yang L, Liu Q, Zhang X, Liu X, Zhou B, Chen J, et al. DNA of neutrophil extracellular traps promotes cancer metastasis via CCDC25. Nature. 2020; 583(7814): 133-8.

97. Liu R, Zhao E, Wang F, Cui H. CCDC25: precise navigator for neutrophil extracellular traps on the prometastatic road. Signal Transduct Target Ther. 2020; 5: 162. doi: 10.1038/s41392-02000285-6.

98. Erpenbeck L, Schön MP. Neutrophil extracellular traps : protagonists of cancer progression? Oncogene. 2017; 36(18): 2483-90. 
99. Rivera-Franco MM, Leon-Rodriguez E, Torres-Ruiz JJ, GómezMartín D, Angles-Cano E, de la Luz Sevilla-González M. Neutrophil extracellular traps associate with clinical stages in breast cancer. Pathol Oncol Res. 2020; 26(3): 1781-5.

100. Martins-Cardoso K, Almeida VH, Bagri KM, Rossi MID, Mermelstein CS, König S, et al. Neutrophil extracellular traps (Nets) promote pro-metastatic phenotype in human breast cancer cells through epithelial-mesenchymal transition. Cancers (Basel). 2020; 12(6): 1542. doi: $10.3390 /$ cancers 12061542.

101. Zhu B, Zhang X, Sun S, Fu Y, Xie L, Ai P. NF-кB and neutrophil extracellular traps cooperate to promote breast cancer progression and metastasis. Exp Cell Res. 2021; 405(2):112707. doi: 10.1016/j. yexcr.2021.112707.

102. Khan U, Chowdhury S, Billah MM, Islam KMD, Thorlacius H, Rahman M. Neutrophil extracellular traps in colorectal cancer progression and metastasis. Int J Mol Sci. 2021; 22(14): 7260. doi: 10.3390/ijms22147260.

103. Yang L, Liu LU, Zhang R, Hong J, Wang Y, Wang J, et al. IL-8 mediates a positive loop connecting increased neutrophil extracellular traps (NETs) and colorectal cancer liver metastasis. J Cancer. 2020; 11(15): 4384-96.

104. Zhang Y, Wang C, Yu M, Zhao X, Du J, Li Y, et al. Neutrophil extracellular traps induced by activated platelets contribute to procoagulant activity in patients with colorectal cancer. Thromb Res. 2019; 180: 87-97.

105. Richardson JJR, Hendrickse C, Gao-Smith F, Thickett DR. Neutrophil extracellular trap production in patients with colorectal cancer in vitro. Int J Inflam. 2017; 2017: 4915062. doi: 10.1155/2017/4915062.

106. Van der Bij GJ, Oosterling SJ, Beelen RHJ, Meijer S, Coffey JC, van Egmond $\mathrm{M}$. The perioperative period is an underutilized window of therapeutic opportunity in patients with colorectal cancer. Ann Surg. 2009; 249(5): 727-34.

107. Richardson JJR, Hendrickse C, Gao-Smith F, Thickett DR. Characterization of systemic neutrophil function in patients undergoing colorectal cancer resection. J Surg Res. 2017; 220: 4108.e1.

108. Tohme S, Yazdani HO, Al-Khafaji AB, Chidi AP, Loughran P, Mowen $\mathrm{K}$, et al. Neutrophil extracellular traps promote the development and progression of liver metastases after surgical stress. Cancer Res. 2016; 76(6): 1367-80.

109. Hahn J, Knopf J, Maueroder C, Kienhofer D, Leppkes M, Herrmann M. Neutrophils and neutrophil extracellular traps orchestrate initiation and resolution of inflammation. Clin Exp Rheumatol. 2016; 34(4 Suppl 98): 6-8.
110. Saffarzadeh M. Neutrophil extracellular traps as a drug target to counteract chronic and acute inflammation. Curr Pharm Biotechnol. 2018; 19(15): 1196-202.

111. Masucci MT, Minopoli M, Del Vecchio S, Carriero MV. The emerging role of neutrophil extracellular traps (NETs) in tumor progression and metastasis. Front Immunol. 2020; 11: 1749. doi: 10.3389/ fimmu.2020.01749.

112. Heuer A, Stiel C, Elrod J, Königs I, Vincent D, Schlegel P, et al. Therapeutic targeting of neutrophil extracellular traps improves primary and secondary intention wound healing in mice. Front Immunol. 2021; 12: 614347. doi: 10.3389/fimmu.2021.614347.

113. Wang H, Wang C, Zhao M, Chen M. Neutrophil extracellular traps can activate alternative complement pathways. Clin Exp Immunol. 2015; 181(3): 518-27.

114. Schreiber A, Rousselle A, Becker JU, Von Mässenhausen A, Linkermann A, Kettritz R. Necroptosis controls NET generation and mediates complement activation, endothelial damage, and autoimmune vasculitis. Proc Natl Acad Sci. 2017; 114(45): E961825.

115. Watanabe-Kusunoki K, Nakazawa D, Ishizu A, Atsumi T. Thrombomodulin as a physiological modulator of intravascular injury. Front Immunol. 2020; 11: 575890. doi: 10.3389/ fimmu.2020.575890.

116. Kajioka H, Kagawa S, Ito A, Yoshimoto M, Sakamoto S, Kikuchi S, et al. Targeting neutrophil extracellular traps with thrombomodulin prevents pancreatic cancer metastasis. Cancer Lett. 2021; 497: 1-13. doi: 10.1016/j.canlet.2020.10.015.

117. Zhu T, Zou X, Yang C, Li L, Wang B, Li R, et al. Neutrophil extracellular traps promote gastric cancer metastasis by inducing epithelial-mesenchymal transition. Int J Mol Med. 2021; 48(1): 127. doi: 10.3892/ijmm.2021.4960.

118. Shimomura Y, Suga M, Kuriyama N, Nakamura T, Sakai T, Kato $\mathrm{Y}$, et al. Recombinant human thrombomodulin inhibits neutrophil extracellular trap formation in vitro. J intensive care. 2016; 4: 48. doi: 10.1186/s40560-016-0177-9.

119. Jain S, Pitoc GA, Holl EK, Zhang Y, Borst L, Leong KW, et al. Nucleic acid scavengers inhibit thrombosis without increasing bleeding. Proc Natl Acad Sci. 2012; 109(32): 12938-43.

120. Yang D, Liu J. Neutrophil extracellular traps: a new player in cancer metastasis and therapeutic target. J Exp Clin Cancer Res. 2021; 40(1): 233. doi: 10.1186/s13046-021-02013-6.

121. Mutua V, Gershwin LJ. A review of neutrophil extracellular traps (NETs) in disease: potential anti-NETs therapeutics. Clin Rev Allergy Immunol. 2021; 61(2): 194-211. 